\title{
Developing Virtual Actors Inspired by Real Actors' Practice
}

\author{
Rogerio E. da Silva \\ Universidade do Minho, Portugal and \\ UDESC, Brazil \\ rsilva@joinville.udesc.br
}

\author{
Manuel F. dos Santos \\ Universidade do Minho, Portugal \\ mfs@dsi.uminho.pt
}

\author{
Ido A. Iurgel \\ Universidade do Minho and \\ Centro de Computação Gráfica, Portugal \\ ido.iurgel@dsi.uminho.pt
}

\begin{abstract}
Autonomous Digital Actors represent the next step in animating 3D characters. How to create such virtual actors remains an open question. This work presents and discusses an agent architecture derived from how real actors learn their art from acting schools. The knowledge base relies on a rule-based system where each rule encodes explicit knowledge into a classification vector which allows agents to suggest performances even for unforeseen situations, and to adjust their suggestions to match the animator's preferences.
\end{abstract}

Keywords-Virtual Actors; Autonomous Digital Actors; Authoring Believable Characters; Computer Animation

\section{INTRODUCTION}

Perlin \& Seidman have already foreseen that " $3 D$ animation and gaming industry will soon be shifting to a new way to create and animate $3 D$ characters, and that rather than being required to animate a character separately for each motion sequence, animators will be able to interact with software authoring tools that will let them train an Autonomous Digital Actor (ADA) how to employ various styles of movement, body language, techniques for conveying specific emotions, best acting choices, and other general performance skills" [1].

Though the term "virtual actor", and analogous terms as "autonomous digital actor" or "synthespian" were already employed by other authors, a systematic incorporation of real actors' practice into formal models is still missing. Real actors learn a series of general purpose techniques applicable to all kinds of roles. Once an actor masters these techniques, it becomes possible to apply them to several different role contexts.

This work reviews the literature on the art of acting and sketches a computational model that allows the implementation of real acting practices. An extensive review of acting schools and script analysis techniques is presented in section III. Then, derived from this study, in section IV we develop a set of requirements for virtual actors; in section $\mathrm{V}$ we sketch an agent architecture that implements these requirements. The conclusions are presented in section VI.

\section{RELATED WORKS}

There are several works that deal with role playing in autonomous characters [2], [3], [4], [5], but their goal was to develop agents capable of performing a single specific role (and only that one). Another important project related to designing characters capable of autonomously interpreting a situation and deriving proper actions is SAIBA [6]. This project aims at "unify key interfaces in the multi-modal behavior generation process" through the introduction of the Behavior Markup Language (BML) that describes "human nonverbal and verbal behavior in a manner independent of the particular realization (animation) method used".

\section{WHAT DO REAL ACTORS DO?}

There are two important lessons that an apprentice actor must learn:

- Performance Skills: how to use his/her body (arms, legs, face, voice, etc.) to better tell a story. There are several acting schools (see section III-A) that suggest their own list of skills.

- Script Analysis: appart from studying performance, he/she needs to learn how to interpret the role and which kind of information is important to extract from the script to understand his/her character.

\section{A. Acting Schools}

1) Constantin Stanislavski: a Russian actor and director, was a legendary acting teacher whose lessons are still today being followed by acting students worldwide [7], [8], [9]:

- A performance without emotions looks artificial.

- An actor should learn how to relax his/her muscles to foster believable movements while performing.

- A performance should be explained as objectives, obstacles and actions.

- An actor should recall his/her own previous experiences and the emotions involved in those (not trying to mimic others), which should allow him to retrieve specific behaviors if needed.

- An actor should start deciding how to perform 'from inside' (role character's point of view) and avoid, by all means, overacting (including too much acting details).

- Being able to adjust the actor's performance regarding changes of other actors or the surrounding environment.

2) The Method: Lee Strasberg, a former student of Stanislavski, and co-founder of Actor's Studio, developed "The Method" that relies on the following fundaments: 
- Following Stanislavski, Strasberg also stresses the importance of the preparation before acting including being relaxed and focused for believability.

- An actor should recall objects and sensory experiences, for instance, how were the sensations when drinking a cup of coffee (considering all five senses).

- Unlike sense memory, emotion memory tries to recall emotional experiences from the actor's past, instead of trying to force a reaction or emotion.

- An actor should know how to build physically and psychologically a character, using both the sense and emotion memory for that.

- Method actors should fully understand their characters in order to perform them believably. Therefore, they are guided to 'answer' questions like "who is the character"? "what does he want"? "why does he want it"? "how is he planning to achieve his/her goals"?

3) Other Schools: Appart from Stanislavski and Strasberg methods, other acting teachers (often followers of these two) have developed or adapted their own acting methods. Two well known schools are the Meisner method and the Adler method [9].

- Sanford Meisner insights:

- An actor should perform as if he were living in the real world.

- A performance should be understood as a series of independent interconnected moments.

- The power of imagination is a much more reliable source than sense memory and should be used instead.

- Stella Adler maintains that:

- Every performed action must have a justification, otherwise it should be avoided.

- Performances are representations of observations of the real world.

- To fully understand his/her character, an actor should exhaustively study the script.

- To give 'size' to a character, an actor should rehearse his/her body and voice.

\section{B. Script Analysis}

There is a consensus among acting teachers that an actor should study his/her role character in depth to prepare himself for his/her part. Figure 1 summarize important aspects of studying a script [10], [11].

\section{What Virtual ACtORS ARE ExPECTED TO DO?}

It is our understanding that a virtual actor is an analogy to a real actor, which autonomously, and by its independent interpretation of the situation, can perform its role according to a given script, as part of a story.

An ADA should be able to perform (at least) the following steps:

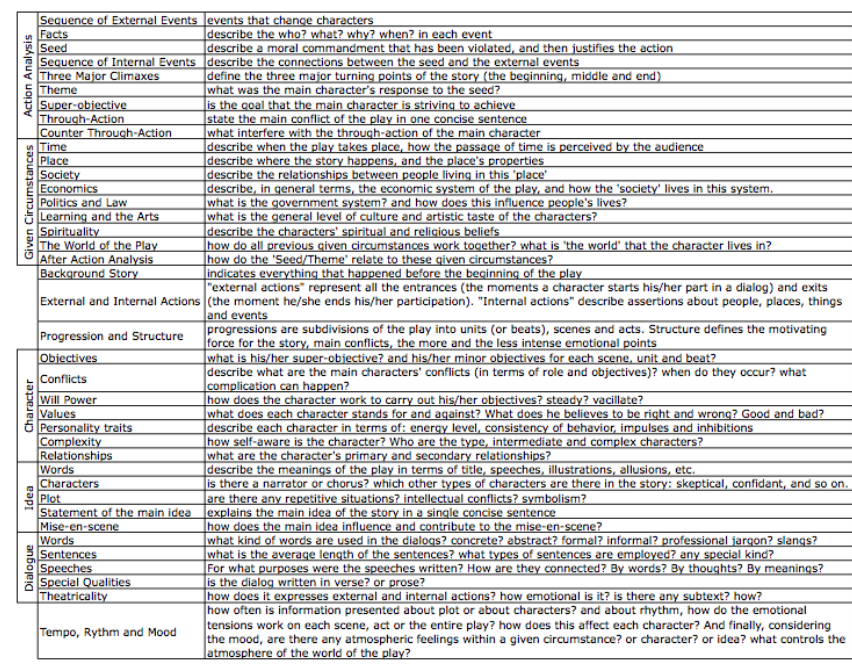

Figure 1. Main Aspects of a Script Analysis

1) Interpretation of script units: A script unit represents one performable action of a character in a story like, for instance, speak or look-to. To allow ADAs to proper interpret a particular script unit, it can be annotated with tags that help actors deciding how to suggest acting performances for them.

2) Acting Performance Suggestion: Suggesting performances means, considering the interpretation made for a script unit and his knowledge about the script, to select a preferred action from a list of plausible ones.

3) Acting Performance: converts each action into animation commands for an animation engine of choice.

4) Accepting Guidance: the animator working as a director, guides all virtual actors on how to improve their suggestions (reinforcement learning).

In a sense, the first three steps correlate with the architecture proposed by Cassell et al for BEAT [12], though it significantly differs in terms of the implementation of each of them.

\section{Developing Virtual Actors}

\section{A. Virtual Actors Architecture}

Our virtual actors were designed as two sets of states: inner states represent any emotional state they can assume while enacting an unit, outer states represent the actual physical action that needs to be performed, for instance "display fear".

The proposed agent architecture (Figure 2) is composed of three sub-modules: the script unit annotation module is responsible for helping the animator to add special tags (e.g. [13]) into each script unit under performance. These tags work as interpretations of the given unit and help virtual actors to decide how it should be performed. Next, the behavior suggestion module combines the tags with 
knowledge from knowledge bases (section V-B), and outputs an acting suggestion by take into consideration three criteria: the acting style (exaggeration or restrained performance); the actors' interaction driving force (cooperation or competition) and the individual performance (list of inner and outer states for the ADA).

Finally, the behavior scheduling module combines the action with timing data from a Text-to-Speech (TTS) software to produce the final suggestion that is then sent as a timeline to the animation engine of choice.

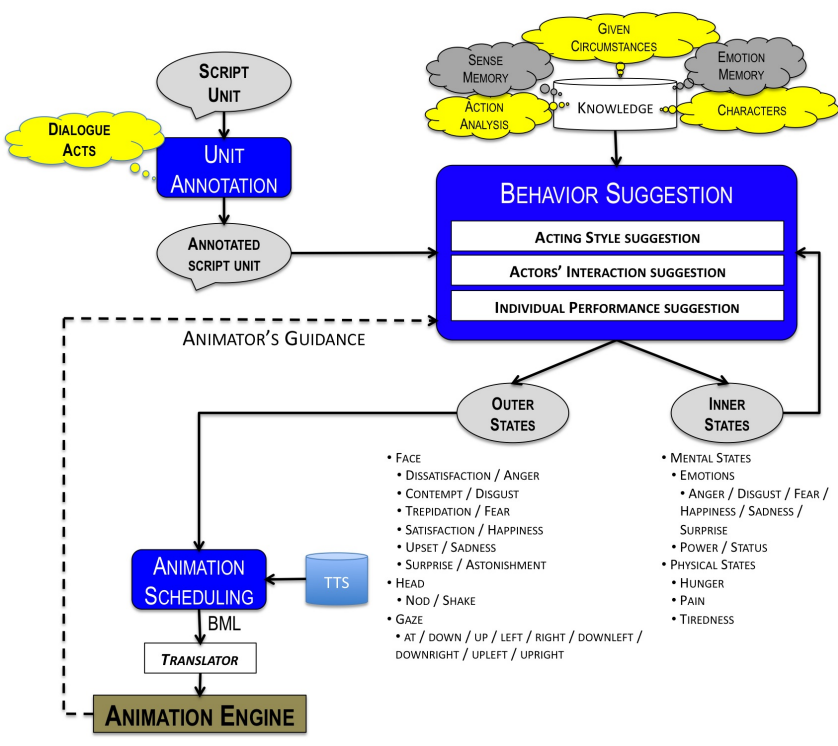

Figure 2. Virtual Actor Architecture

\section{B. Knowledge Representation for Virtual Actors}

What we want to do is to be able to train virtual actors in a way that resembles how real actors learn to perform.

We have selected, from the studies made on acting (section III), to focus on three main aspects that are essential for novice actors to learn ${ }^{1}$ : emotion memory, sense memory and script analysis.

- Emotion Memory: the actor memorizes appropriate emotional responses and the context in which it has occurred so, in the future, the actor can try to replicate the same inner state in similar situations.

- Sense Memory: analogously to emotion memory, previous situations that resulted in physical reactions (e.g. pain) are stored for future reference.

- Script Analysis: collects a series of information from the script being performed: characterization, given circumstances and the action analysis. This information enables the virtual actors to interpret the current context

\footnotetext{
${ }^{1}$ All physical aspects of acting like muscle relaxation, and concentration, have been disregarded from our model because they do not easily apply to animated characters
}

they are inserted into and decide on adequate ways of acting:

- Characterization: is a collection of facts that describes the role character being played by the ADA. The current version supports the following attributes:

1) Personal data: age, gender

2) Objective: search_for_stability, search_for_excitement, indifference

- Given Circumstances: is a collection of facts that describes the current context of the scene being played. Supported values are:

* Time: day, night, present, past and future

* Place: home, work, street

- Action Analysis: is the quick analysis made on the script to be enacted. Currently the only supported two elements are:

* Theme: only quarreling_scene for now

* Dialogues: is composed of the list of annotated script units described by the tuple [speaker name, listener names, dialog act tag]

1) Designing a Rule-based System from Classification Vectors: The problem with rule-based systems is its perfect matching selection criteria, which means that, given a desired context, only those rules that perfectly match will be considered. This means that we would need an enormous list of rules to cover all possible scenarios; otherwise, the actor would get stucked by unforeseen situations. Furthermore, we would produce actors that always suggest the same performance for a reoccurring context.

What is desired is that virtual actors constantly update their suggestions (to match animator's preferences, for instance) and to come up with a suggestion even for unforeseen situations.

Our solution is based on a textming technique called "classification vectors". A classification vector is a list of probabilities that describes how a given context is related to a given class. E.g., consider that a context could be classified in $n$ different classes, so its classification vector is defined as $<\operatorname{Prob}_{1}, \mathrm{Prob}_{2}, \ldots, \operatorname{Prob}_{n}>$ where $\mathrm{Prob}_{i}$ indicates the probability of the given context being related to the class $i$.

Our solution is an extension of this idea, where a rule is coded into a class vector, while each position indicates one attribute from one of the aspects of acting schools presented before, as illustrated in Figure 3.

Using this representation it is possible to determine how similar two vectors are by calculating the linear distance between them: $\operatorname{distance}(\vec{A}, \vec{B})=\sum_{i=1}^{n} \vec{A}_{i}-\vec{B}_{i}$. So, considering that a given situation (that needs to be enacted) is equally represented as a vector, one could compare it against all the rules in ADA's knowledge base. The minimal distance indicates the most similar rule. In cases where more than one 


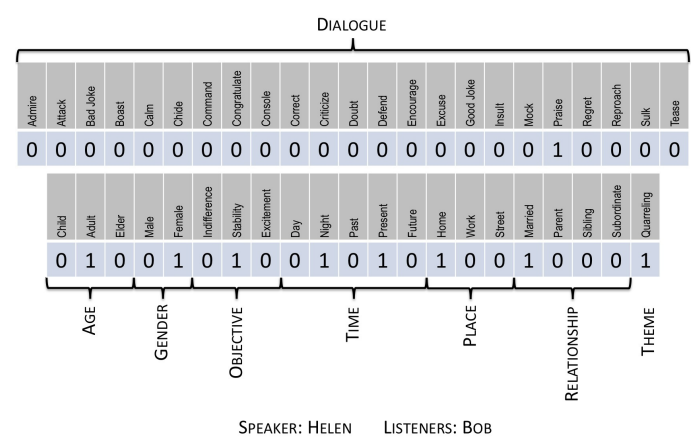

Figure 3. Partial representation of a context clause of a rule

rule has the same distance, other criteria can be employed to select the rule for action.

One possibility is to associate a support level (number of times the rule has been selected for acting) and a confidence level (number of times that the animator did not object to the use of the rule) to each rule. So, the ratio $=\frac{\text { confidence }}{\text { support }}$ gives us feedback about the animator's preferences, and after some time all actors would have been calibrated to match them, resulting in better suggestions (reinforcement learning).

\section{CONCLUSIONS}

Using autonomous digital actors (also called virtual actors) to assist the creation of an animation film is expected to be the next step in authoring believable characters for computer animation.

This work aims at contributing to this development with a report on how real actors learn to act and how they apply this knowledge and skills to perform a given role of a film script. We maintained that, for a virtual actor to mimicry this process, it should possess at least the following skills: interpreting each unit of a script, inferring how a person should feel and act in each given situation, suggesting appropriate behaviors (both in terms of feelings and actions) and, after performing its first suggestions, to adapt its performance to match the animator's further instructions.

We proposed an agent architecture that relies on a rulebased system where rules encode extracted knowledge from approaches used by real actors to learn and prepare for acting. Our system adapts classification vectors commonly used by textmining applications. This vector representation allows us to produce actors capable of suggesting performances even for unforeseen situations and to adapt themselves by learning the animator preferences through reinforcement learning. That is particularly aggraveted by the large domain and rule set that results from an implementation of real acting knowledge, as suggested in this paper.

\section{ACKNOWLEDGEMENTS}

This research is partly funded by the Portuguese Science and Research Foundation FCT (Fundação para a Ciência e a Tecnologia), under reference PTDC/EIA/69236/2006, VirtualActor project.

\section{REFERENCES}

[1] K. Perlin and G. Seidman, "Autonomous digital actors," in Motion in Games, 2008, pp. 246-255. [Online]. Available: http://dx.doi.org/10.1007/978-3-540-89220-5_24

[2] R. S. Aylett, S. Louchart, J. Dias, A. Paiva, and M. Vala, "FearNot! - an experiment in emergent narrative," in Intelligent Virtual Agents. Springer, 2005, pp. 305-316. [Online]. Available: http://dx.doi.org/10.1007/11550617_26

[3] S. Baldes, P. Gebhard, M. Kipp, M. Klesen, P. Rist, T. Rist, and M. Schmitt, "The interactive CrossTalk installation: MetaTheater with animated presentation agents," in Proceedings of the Workshop on "Lifelike Animated Agents" held in conjunction with the Seventh Pacific Rim International Conference on Artificial Intelligence, 2002, pp. 9-15.

[4] M. Mateas and A. Stern, "Façade, an experiment in building a fully-realized interactive drama," in Game Developers Conference, San Jose, CA., Mar. 2003.

[5] B. Hayes-roth, R. V. Gent, and D. Huber, "Acting in character," in Autonomous Agents and Multi-Agent Systems, 1997, pp. 92-112. [Online]. Available: http: //citeseerx.ist.psu.edu/viewdoc/summary?doi=10.1.1.57.2156

[6] H. Vilhjálmsson, N. Cantelmo, J. Cassell, N. E. Chafai, M. Kipp, S. Kopp, M. Mancini, S. Marsella, A. Marshall, C. Pelachaud, Z. Ruttkay, K. Thórisson, H. van Welbergen, and R. van der Werf, "The behavior markup language: Recent developments and challenges," in Intelligent Virtual Agents, 2007, pp. 99-111. [Online]. Available: http: //dx.doi.org/10.1007/978-3-540-74997-4_10

[7] C. Stanislavski, An Actor Prepares. Methuen Drama, 1937.

[8] —, Building A Character. Methuen Drama, 1950.

[9] Acting-School-Stop.com, "Acting techniques," http://www.acting-school-stop.com/index.html, Apr. 2010. [Online]. Available: http://www.acting-school-stop.com/ index.html

[10] J. Thomas, Script Analysis for Actors, Directors and Designers, 4th ed. Focal Press, 2009.

[11] C. S. Waxberg, The Actor's Script: Script Analysis for Performers, 1st ed. Heinemann Trade, 1998.

[12] J. Cassell, H. H. Vilhjalmsson, and T. Bickmore, "BEAT: the behavior expression animation toolkit," in Proceedings of the 28th annual conference on Computer graphics and interactive techniques. ACM, 2001, pp. 477-486. [Online]. Available: http://portal.acm.org/citation.cfm?id=383315\&dl=

[13] P. Gebhard, M. Klesen, and T. Rist, "Adding the emotional dimension to scripting character dialogues," Proc. of the 4th International Working Conference on Intelligent Virtual Agents (IVA'03), Kloster Irsee, vol. 2003, pp. 48 -56, 2003. [Online]. Available: http://citeseerx.ist.psu. edu/viewdoc/summary?doi=10.1.1.89.9891 